\title{
Procédé Amélioré De Conservation Et De Stabilisation Du Fromage Peuhl Par L'effet Combiné Du Traitement Thermique Et Du Conditionnement Sous Vide
}

\author{
Dossou Joseph \\ Montcho Jules Karel \\ Londji Serge \\ Atchouké G. Donald Laurent \\ Odjo Sylvanus
}

Département de Nutrition et Sciences Alimentaires, Faculté des Sciences

Agronomiques, Université d'Abomey-Calavi, 01 BP 526 Cotonou, Bénin

doi: 10.19044/esj.2016.v12n36p189 URL:http://dx.doi.org/10.19044/esj.2016.v12n36p189

\begin{abstract}
This study focused on improving the conservation of traditional Fulani cheese (Wagashi) from Benin by using the combined effect of thermic dehydration and vacuum packaging. In order to increase the shelf life and cheese stability, some experiments were undertaken using heat treatment at 60 ${ }^{\circ} \mathrm{C}$ for 4 hours and vacuum packaging. The treated cheeses had a water content ranging from 32 to $35 \%$ and showed a reduced microbial load (102 CFU/g for the total aerobic mesophyl germs and $101 \mathrm{CFU} / \mathrm{g}$ for lactobacilli, yeasts and moulds. The cheeses nutritional and sensory quality was also preserved after treatment. Microbiological and physicochemical analyses carried out periodically on processed and stored cheeses in vacuum packaging showed these cheeses are stable for about 60 days, both under refrigeration $\left(4-5^{\circ} \mathrm{C}\right)$ and ambient temperature $\left(28-32^{\circ} \mathrm{C}\right)$. These results were confirmed by sensory analyses. However, over a two month's period, the panelists preferred white cheeses than red colored cheeses stored under vacuum. The economic analysis performed on the mini-factory basis with a cheese processing capacity of 12000 liters milk per year, showed that heat treated cheese stored in vacuum packaging costs 55.35 FCFA a piece of $50 \mathrm{~g}$ (net weight) with a selling price of 150 FCFA giving a net profit of $63.1 \%$ and break-even point by $54.15 \mathrm{~kg}$ of cheese production.
\end{abstract}

Keywords: Milk, Wagashi, Drying, Sensory test, Costs, Profitability 


\section{Resume}

La présente étude a porté sur la mise au point et le test d'un procédé amélioré de conservation du fromage peulh (Wagashi) par utilisation combinée de la déshydratation thermique et du conditionnement en emballage sous vide. Des essais de prolongation de la durée de conservation et de la stabilité du fromage peulh ont été entrepris en utilisant le traitement thermique déshydratant à 60 ${ }^{\circ} \mathrm{C}$ pendant 4 heures et le thermo soudage sous vide en emballage plastique gaufré. Les fromages ainsi traités ont une teneur en eau variant entre 32 et $35 \%$ et présentent des charges microbiennes réduites de l'ordre de $102 \mathrm{CFU} / \mathrm{g}$ pour les germes aérobies mésophiles totaux et de l'ordre de $101 \mathrm{CFU} / g$ pour les lactobacilles, les levures et les moisissures. La qualité nutritionnelle et sensorielle des fromages a été préservée après le traitement. Les analyses microbiologiques et physico-chimiques effectuées périodiquement sur les fromages traités et conservés en emballage sous vide ont révélé que les fromages restent stables pendant environ 60 jours, aussi bien à la température de réfrigération $\left(4-5^{\circ} \mathrm{C}\right)$ qu'à la température ambiante entre 28 à $32^{\circ} \mathrm{C}$. Les analyses sensorielles ont confirmé ces résultats, mais les dégustateurs ont marqué leur préférence pour les fromages blancs par rapport aux fromages colorés en rouge conservés sous vide au-delà de deux mois. L'analyse économique de la production de fromage traité et conservé sous vide, effectuée sur la base d'une capacité de transformation de 12000 litres de lait par an, a permis d'évalué le coût de production d'un morceau de $50 \mathrm{~g}$ net de fromage à 55,35 FCFA, le prix de vente à 150 FCFA.

Mots-Clefs : Lait, Wagashi, Sechage, Test Sensoriel, Couts, Rentabilite.

\section{Introduction}

Connu sous l'appellation courante de Wagashi au Benin et plus spécifiquement de Gassiré en langue locale Fulfudé, le fromage peulh est un fromage à pâte molle de haute valeur nutritionnelle, obtenu par coagulation à chaud du lait frais entier, sous l'action de la calotropaïne, une enzyme végétale de Calotropis procera (Dossou, 2015). Ce fromage est très apprécié et fortement demandé par les populations du Bénin, du Togo, du Nigéria et d'autres pays de la sous-région ouest-africaine (Dossou et al, 2006). Dans ces pays, il est consommé fréquemment en remplacement de la viande et du poisson dans divers plats alimentaires (Kees, 1996). Dans ces régions tropicales chaudes où le lait se valorise mal à cause de sa forte périssabilité due à la température ambiante généralement élevée $\left(30\right.$ à $\left.45^{\circ} \mathrm{C}\right)$, le fromage peulh représente une forme alternative intéressante de conservation du lait, puisqu'il en préserve la valeur économique et alimentaire. En dépit de cette importance économique et nutritionnelle, le conditionnement et la 
conservation du fromage présentent de nombreuses contraintes, comme par exemple la non efficacité des méthodes traditionnelles de conservation du fromage, se traduisant par une détérioration précoce du produit dans le circuit de commercialisation, l'exposition du fromage traditionnel à l'air libre; ce qui en augmente les risques de contaminations microbienne et chimique, l'absence d'emballage approprié pour le fromage limitant ses aptitudes à la conservation, au transport et à la commercialisation sur le marché local et à l'exportation (Dossou, 2004). Outmani (2009) rapporte que les fromagères du Nord-ouest du Bénin sont confrontées à des difficultés de commercialisation dues, entre autres, à la mauvaise présentation du Wagashi. Dans le but d'allonger la durée de conservation du fromage peulh, des travaux ont été menés sur sa stabilisation par traitement chimique et sur le conditionnement du Wagashi (Egounléty, 1982). Il résulte de ces travaux que le traitement du fromage avec l'acide propionique à $10 \%$ et l'acide sorbique à $0,1 \%$ prolonge la durée de sa conservation au-delà de 20 jours, alors que les échantillons témoins présentent des signes visibles de détérioration seulement après une période d'entreposage de 9 jours. Ces traitements réduisent également la charge microbienne du fromage. Cependant, une évaluation organoleptique a démontré que l'acide sorbique confère un goût amer au fromage, l'acide propionique un goût sucré ; ce qui pourrait ainsi affecter l'acceptabilité du produit. Ces études, qui ont été conduites essentiellement au laboratoire, n'ont pas d'incidences améliorantes sur la technologie traditionnelle de fabrication de fromage (Dossou et al., 2006). De plus, les travaux plus récents de Kèkè (2005) ayant porté sur l'utilisation d'une souche lyophilisée de Lactobacillus plantarum en vue de conserver le Wagashi n'ont pas abouti à des résultats satisfaisants car, ce traitement donne un goût acide au produit. De nouvelles recherches sur cette problématique s'avèrent donc indispensables. La présente étude vise à améliorer la durée de conservation du fromage peulh par la mise en œuvre d'un procédé technologique combinant le traitement thermique et l'emballage sous vide.

\section{Matériel et méthodes}

L'approche méthodologique globale utilisée dans la présente étude consiste à évaluer l'effet du vide sur la qualité du fromage peulh. À cet effet, deux lots d'échantillons de fromage ont été fabriqués et conditionnés sous vide. Chacun des lots est divisé en deux autres lots qui sont mis en conservation, l'un à la température ambiante à $28-32{ }^{\circ} \mathrm{C}$, l'autre à la réfrigération à $4-5^{\circ} \mathrm{C}$. L'évolution de la qualité de ces lots de fromage a été suivie sur une période de deux mois à travers les analyses de leurs paramètres physico-chimiques et microbiologiques ainsi que des tests de dégustation. 


\section{Matériel}

\section{Matière première et ingrédients utilisés}

La matière première regroupe le lait de vache, le coagulant végétal et le colorant $\mathrm{du}$ fromage. Le lait utilisé pour la production du fromage expérimental est un mélange de lait de vache de différentes races locales, notamment les races Borgou et Lagunaire. Il a été collecté dans les campements peulh situés dans la zone péri urbaine de Cotonou et d'AbomeyCalavi. Deux lots de quarante (40) litres de lait ont été utilisés, l'un pour la fabrication des fromages colorés en rouge, l'autre pour la fabrication des fromages blancs. Les feuilles et tiges de Calotropis procera récoltées sur le Campus de l'Université d'Abomey-Calavi ont été triturées en ajoutant une petite quantité de lait frais et l'extrait liquide obtenu a servi de coagulant pour la fabrication du fromage. La panicule de Sorghum vulgaris a été achetée au marché d'Abomey-Calavi et infusée à chaud pour obtenir l'extrait aqueux ayant servi à la coloration en rouge des fromages. Le sel de cuisine et la potasse ont été utilisés lors de la coloration des fromages peulh. Le sel permet de donner un goût agréable au fromage. La potasse permet la fixation du colorant.

\section{Matériel de mise en emballage sous vide}

Le matériel d'emballage utilisé est de deux types : les sachets gaufrés de dimension $30 \mathrm{~cm}$ X20 cm découpés en 8 morceaux et thermosoudés. Réduits ainsi à la taille des fromages de petites pièces de 7 à $10 \mathrm{~cm}$ de diamètres environ, ils sont ensuite introduits dans d'autres sachets en polyéthylène haute densité et l'ensemble, étanchéifié, a été soumis à une stérilisation dans un Bain Marie à $105{ }^{\circ} \mathrm{C}$ pendant 15 minutes. De même, les gants en latex et la fourchette qui ont servi au prélèvement et à l'ensachage des fromages ont été stérilisés dans le Bain Marie.

Les équipements utilisés lors du processus de mise en conservation sous vide se composent essentiellement comme suit: une thermosoudeuse de marque Calor a permis la soudure des sachets gaufrés après leur découpage en des dimensions voulues ; un séchoir de type Heraeus est utilisé pour le traitement thermique appliqué aux fromages ; le premier lot d'échantillons de fromage mis sous réfrigération a été conservé dans un réfrigérateur de marque Arthur Martin, tandis qu'une caisse d'entreposage de fromage en bois de dimension $45 \mathrm{~cm}$ x $32 \mathrm{~cm} \mathrm{x} 38,5 \mathrm{~cm}$ a servi à garder le deuxième lot de fromages emballés sous vide et conservés à la température ambiante. Le conditionnement des fromages a été réalisé à l'aide d'un appareil de mise sous vide de marque Besser. Il s'agit d'une machine semi-automatique destinée à l'usage domestique. 


\section{Méthodes}

\section{Procédé de fabrication et de conservation des fromages sous vide}

Les différents types de fromages ont été préparés selon la méthode rapportée par Dossou et al., (2006). Cette méthode est définie suivant le diagramme de la figure 1 .

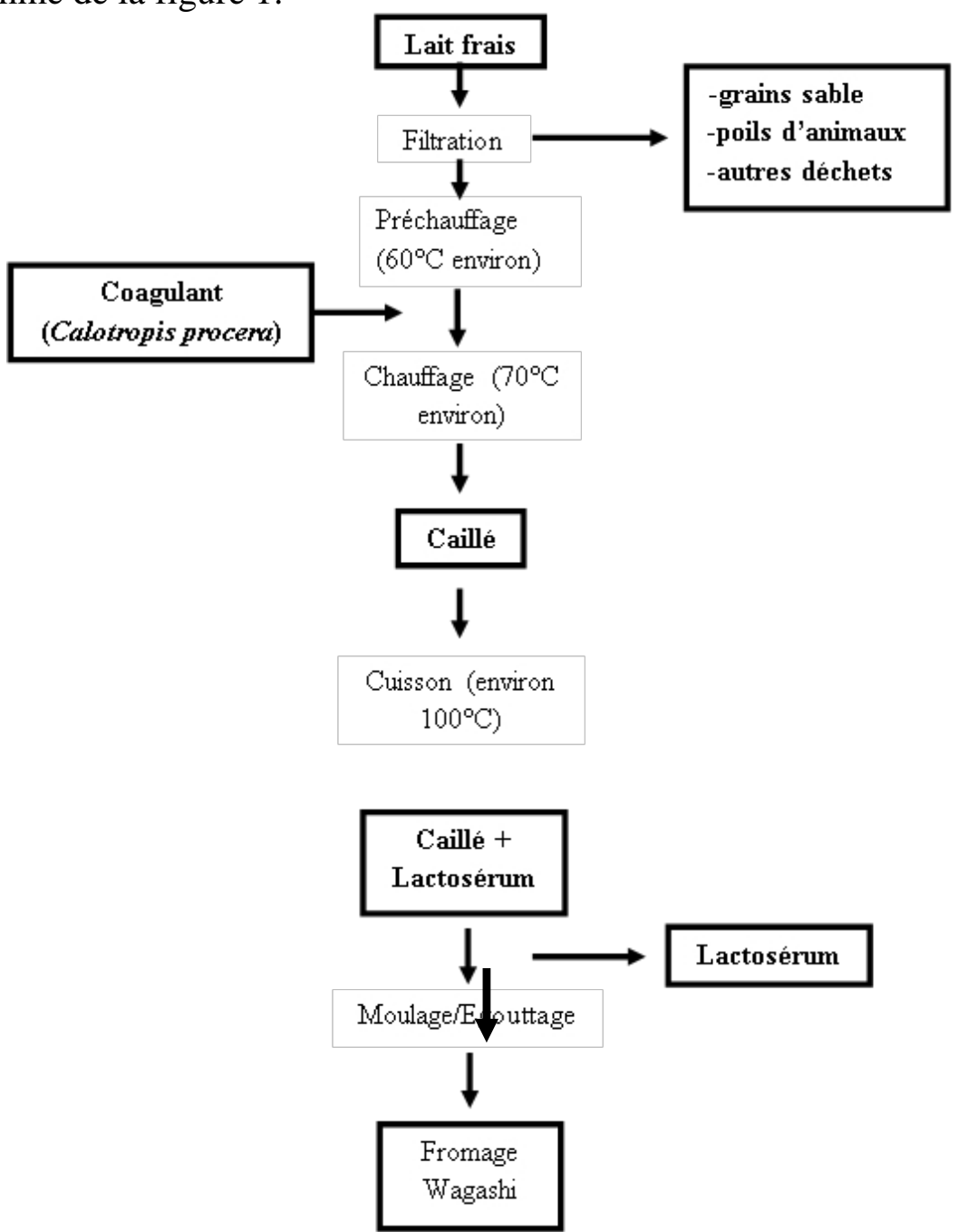

Figure 1 : Diagramme technologique de production du fromage Wagashi (Dossou et al., 2006)

Les fromages ont été soumis à un traitement thermique déshydratant avant leur mise sous vide, avec pour objectif, la réduction de leur teneur en eau et de leur charge microbienne. Ce séchage a été réalisé à $60^{\circ} \mathrm{C}$ pendant 4 heures de temps dans un séchoir de type Heraeus. Les fromages déshydratés et pasteurisés ont été emballés sous vide dans les sachets gaufrés en deux lots 
différents. Chaque lot est constitué de fromages rouges et de fromages blancs emballés séparément. Après avoir fait le contrôle de la bonne qualité du thermosoudage, de l'absence de poche d'air ou d'impuretés, les deux lots de fromage emballé ont été rangés pour la conservation, l'un à la température ambiante $\left(28\right.$ à $\left.32^{\circ} \mathrm{C}\right)$ dans la caisse et l'autre à la température de réfrigération (4 à $5{ }^{\circ} \mathrm{C}$ ) dans le réfrigérateur. Les expériences de conservation se sont étendues sur deux mois, au cours desquels la qualité des fromages a été analysée.

Les différentes analyses microbiologiques, physico-chimiques et sensorielles ont été effectuées d'abord au début de l'expérience (T0) puis à une fréquence d'un mois sur les différents types de fromage.

\section{Analyses physico-chimiques des fromages et du lait}

La teneur en eau a été déterminée selon la méthode AA CC 44-15A (AACC, 1984) par séchage à l'étuve à $105{ }^{\circ} \mathrm{C}$, le taux de cendres par incinération au four à $550^{\circ} \mathrm{C}(\mathrm{AOAC}, 1984)$, le $\mathrm{pH}$ et $1^{\prime}$ 'acidité titrable ont été déterminés suivant le protocole de Nout et al. (1989) modifié. La couleur des échantillons a été déterminée à l'aide d'un chromamètre MinoltaChroma CR210 b. Les protéines ont été déterminées par la méthode de Kjeldahl (AOAC, 1984) et les lipides par la méthode de Soxhlet (AOAC, 1984). Les sucres réducteurs ont été dosés par la méthode de Luff-Schoorl. La texture a été évaluée à l'aide d'un texturomètre de marque LF plus (2NOYD instruments) muni d'une sonde de cisaillement triangulaire de type Warner Bratzler. Toutes les analyses ont été faites en deux répétitions. Ces mêmes analyses ont été effectuées sur le lait, excepté la texture, le taux de lipide, la teneur en protéines et la couleur. Le taux de matière grasse du lait a été déterminé suivant la méthode acido-butyrique de GERBER; le taux de protéine du lait par la méthode colorimétrique avec le noir amidon.

\section{Analyses microbiologiques des fromages et du lait}

Les analyses microbiologiques ont pris en compte le dénombrement des germes aérobies mésophiles totaux, des bactéries lactiques, des levures et moisissures et des entérobactéries. Elles ont été faites sur le lait et les fromages, afin d'évaluer la qualité hygiénique de ces produits. Toutes les manipulations ont été réalisées dans des conditions d'asepsie totale. Le dénombrement des germes aérobies mésophiles a été réalisé sur gélose standard pour numération, Plate Count Agar (PCA, oxoid, CM 325, Hampshire, England), selon la norme française V 08-051. Les bactéries lactiques ont été dénombrées sur le milieu Man Rogosa Sharpe (MRS, CM 361, Oxoid, Hampshire, England) contenant 0,1\% (W/V) de natamycine (Delvocid, Gistbrocades, Delft, Netherland). Les levures et les moisissures ont été dénombrées en boîte de Pétri stérile à l'aide du milieu de culture MEA 
(Malt Extract Agar). Le dénombrement des colonies blanches ou colorées, lisses et crémeuses de levures et des moisissures sous forme poudreuse a été effectué après 5 jours d'incubation à $25^{\circ} \mathrm{C}$ selon la norme ISO 7954. Les entérobactéries ont été recherchées selon la méthode décrite par la norme NF ISO 4831. Le dénombrement des entérobactéries (coliformes totaux) a été fait sur la gélose glucosée biliée au cristal violet et au rouge neutre (VRBG, oxoid $\mathrm{CM}$ 485). Pour le comptage, seules les colonies roses à rouges ou violettes avec ou sans halo de précipitation ont été prises en compte.

\section{Analyses sensorielles}

Un panel de 25 consommateurs de fromage peulh, préalablement entraînés pour le test à réaliser, a été formé. Trois séances de dégustation ont été organisées avec l'ensemble du panel à des intervalles réguliers de 30 jours. Chaque série de tests avait pour but d'effectuer une étude comparative des deux types de fromages. Elle a été constituée de tests de différenciation 2 sur $5(2 / 5)$ et d'un test hédonique de classement par ordre de préférence sur une échelle à neuf niveaux où 1 correspond à « Extrêmement désagréable » et 9 à « Extrêmement agréable ». Lorsque le test $2 / 5$ était positif, c'est-à-dire que le nombre de dégustateurs ayant correctement répondu est suffisant par référence aux tables de signification du test de différentiation 2/5 (avec un panel de vingt personnes, il fallait au moins cinq bonnes réponses pour considérer que les produits étaient significativement différents), un test hédonique de classement par ordre de préférence a été effectué avec les dégustateurs ayant perçu des différences. Il a été demandé aux dégustateurs de donner leur appréciation sur la couleur, la texture, l'odeur, le goût, la friabilité et la fermeté pour chaque type de fromage et enfin de faire un choix de préférence entre les deux types de fromages.

\section{Analyse économique}

L'analyse économique de la production de fromage conservé sous vide a été effectuée en tenant compte du coût de la matière première, de la maind'œuvre, de l'amortissement du matériel, des frais de commercialisation et des frais financiers, en vue de calculer le coût de production, le résultat d'exploitation, le seuil de rentabilité de l'activité et la marge nette. Pour un cycle de production à l'échelle artisanale de 40 litres de lait par jour, le coût de la main-d'œuvre a été calculé en considérant 8 heures de travail par jour rémunérées en milieu péri-urbain à 1500 FCFA. Les frais de commercialisation ont été évalués dans l'hypothèse d'une livraison du produit aux revendeuses de fromage peulh au marché Dantokpa (Marché international sis à Cotonou); le prix transport (aller et le retour) du lieu de production au marché Dantokpa pour la livraison étant estimé à 800 FCFA. Le prix du lait en saison pluvieuse est de 500 FCFA et 700 FCFA en saison sèche. Le prix 
moyen du lait considéré dans cette étude est de 600 FCFA. La transformation des 40 litres de lait a nécessité l'achat de $15 \mathrm{~g}$ de panicules de sorgho à 25 FCFA, $30 \mathrm{~g}$ de sel de cuisine à $25 \mathrm{FCFA}, 45 \mathrm{~g}$ de potasse à $25 \mathrm{FCFA}, 200$ FCFA de charbon de bois utilisé comme combustible, 25 litres d'eau estimés à 30 FCFA et la consommation énergétique de $14 \mathrm{~kW}$ estimée à 300 FCFA pour le séchage. Pour une production journalière, 15 sachets gaufrés de dimension $30 \mathrm{~cm}$ x $20 \mathrm{~cm}$ achetés à 76,47 FCFA l'unité sont utilisés. Les amortissements des équipements ont été calculés en divisant le prix à l'achat de ces équipements par leur durée de vie exprimée en année. Le prix de vente des fromages a été estimé en considérant le prix sur le marché des fromages ayant le même poids que le poids frais des fromages expérimentaux séchés. L'estimation a donné 150 FCFA par fromage expérimental présentant un poids moyen égal à $50 \mathrm{~g}$ après séchage et conditionnement sous vide. Enfin, l'analyse économique a été faite en considérant trois cent (300) jours de production par an.

\section{Analyse statistique des données}

Le traitement des données a été réalisé à l'aide du logiciel SPSS 16.0 for Windows sur la base de l'analyse de variance (ANOVA). La comparaison entre les moyennes de deux résultats d'analyses a été effectuée sur la base du test multiple de Duncan. La différence significative a été observée à $\mathrm{P}<0,05$.

\section{Résultats et discussion}

\section{Caractéristiques microbiologiques et physico-chimiques du lait utilisé : Caractéristiques microbiologiques du lait utilisé}

Le lait de vache constitue la principale matière première utilisée pour la fabrication du fromage. L'évaluation de la qualité microbiologique de ce lait a révélé que la charge des germes aérobies mésophiles totaux, des lactobacilles ainsi que celles des entérobactéries sont évaluées à $4,6.10^{7} \mathrm{UFC} / \mathrm{g}, 2,45.10^{5} \mathrm{UFC} / \mathrm{g}$ et $4,2.10^{5} \mathrm{UFC} / \mathrm{g}$ respectivement. Ces différentes charges microbiennes obtenues sont inférieures à celles identifiées dans d'autres travaux dont celui de Kora (2005) qui a révélé des charges de $6,5.10^{7} \mathrm{UFC} / \mathrm{g} ; 1,1.10^{6} \mathrm{UFC} / \mathrm{g}$ et $4,2.10^{5} \mathrm{UFC} / \mathrm{g}$ respectivement pour les germes aérobies mésophiles totaux, les bactéries lactiques et les entérobactéries. Par ailleurs, la valeur moyenne de la charge des germes aérobies mésophiles totaux obtenue pour nos échantillons est élevée par rapport à celle trouvée par Oubari (2004) qui est de 7,42.106 UFC/g, Taybi et al. (2014) qui est de $2.1510^{7} \mathrm{UFC} / \mathrm{g}$ et celle avancée par Hamama et El Mouktafi (1990) qui est de $2.10^{7} \mathrm{UFC} / \mathrm{g}$. Il en est de même pour les résultats obtenus par Labioui et al., (2009) en ce qui concerne les charges des germes aérobies mésophiles totaux $\left(6,38.10^{6} \mathrm{UFC} / \mathrm{g}\right)$, des entérobactéries $\left(2,0.10^{4}\right.$ $\mathrm{UFC} / \mathrm{g})$ et des bactéries lactique $\left(3,5.10^{5} \mathrm{UFC} / \mathrm{g}\right)$ qui sont relativement 
pareilles. De plus, la charge des germes aérobies mésophiles totaux trouvée (4,6.107 UFC /g) dépasse de loin la norme fixée par Directive 92/46/CEE (1992) qui stipule une teneur en germes aérobies mésophiles totaux $<10^{6}$ UFC /g. Cela laisse conclure que la qualité de ce lait n’est irréprochable, et ceci pourrait s'expliquer par le manque d'hygiène aussi bien au niveau de l'animal qu'au niveau du trayeur et du circuit de collecte du lait. Cependant, ces différents résultats obtenus dans la présente étude restent globalement satisfaisants et sont similaires à ceux trouvés par Egounléty et al. (1994) ; Florez et al. (2006) et Sacramento (2008). Pour ces auteurs, dans le lait frais, la charge des germes aérobies mésophiles totaux varient de $1,7 \times 10^{5}$ à $6,8 \times 10^{7} \mathrm{UFC} / \mathrm{g}$, celle des entérobactéries de $4,9 \times 10^{3}$ à $1,9 \times 10^{5} \mathrm{UFC} / \mathrm{g}$ et celle des lactobacilles de $2,7 \times 10^{4}$ à $2,3 \times 10^{6} \mathrm{UFC} / \mathrm{g}$.

\section{Caractéristiques physico-chimiques des laits utilisés}

L'analyse des caractéristiques physico-chimiques du lait ayant servi à la fabrication des fromages blancs et des fromages rouges montre que la qualité physico-chimique du lait utilisé est conforme aux exigences de la norme NF ISO 11816-1 portant sur les teneurs recommandées pour les principaux constituants du lait. En effet, le lait frais utilisé présente une teneur en eau de $85,63 \%$, un $\mathrm{pH}$ de 6,6 , une teneur en protéines de $31,49 \%$, une teneur en lipides de 34,51\% et une acidité titrable de 2,1\%. Des résultats similaires ont été obtenus par Kora (2005) (83,6\%; 6,6; 31,5\% MS ; 34,52\% MS respectivement pour la teneur en eau, le $\mathrm{pH}$, la teneur en protéines et en lipides du lait de vaches de race Borgou) et Dossou et al.(2016) (86,8\% $\pm 0,010$ et 6,5 $\pm 0,057$ respectivement pour la teneur en eau et le $\mathrm{pH}$ du lait de vaches de race Borgou). Par contre, selon les travaux de ces auteurs, des résultats très élevés ont été observés pour la teneur en protéine du lait de vache de race Borgou $(42,70 \pm 0,002 \% \mathrm{MS})$ et pour l'acidité titrable de ce même lait $(15,82$ $\left.\pm 0,000^{\circ} \mathrm{D}\right)$. Par ailleurs, la teneur, en minéraux totaux, en sucres totaux et sucres réducteurs exprimée en base sèche donne respectivement, 1,14\%, 33\% et $18,03 \%$. Ces valeurs sont conformes à celle de la norme NF ISO 11816-1.

\section{Impact du traitement thermique sur la qualité des fromages peulh}

Le séchage a abouti à l'obtention de fromages déshydratés dont les caractéristiques microbiologiques, physico-chimiques et sensorielles diffèrent de celles du fromage frais.

\section{Effet du séchage sur la qualité microbienne des fromages}

Le tableau I présente l'effet du séchage sur la charge microbienne des fromages. Il ressort de l'analyse de ce tableau que, d'une part, les fromages séchés ont une charge microbienne nettement inférieure à celle des fromages frais. La charge des germes aérobies mésophiles totaux, des lactobacilles, des 
entérobactéries et des levures et moisissures des fromages séchés (blancs et rouges) diffère significativement $(\mathrm{p}<0,05)$ de celle des fromages frais (blancs et rouges). Ces résultats montrent une efficacité du barème (temps/température) de traitement thermique appliqué lors de la stabilisation du fromage. D'autre part, il est à noter que parmi les fromages séchés, ceux colorés en rouge possèdent la charge microbienne la plus réduite. La coloration des fromages frais blancs en rouge a donc donné un meilleur résultat, quant à la charge microbienne. De même, les travaux de Amegnoin, (2012) confirme les résultats de la présente étude : les résultats de cet auteur ont conduit à la conclusion selon laquelle le dénombrement de la flore totale, de la flore lactique, des entérobactéries ainsi que celles des levures et moisissures des Wagashi blancs a donné respectivement $4.10^{2} \mathrm{UFC} / \mathrm{g} ; 2.10^{3}$ $\mathrm{UFC} / \mathrm{g} ; 10^{1} \mathrm{UFC} / \mathrm{g}$ et $4.10^{2} \mathrm{UFC} / \mathrm{g}$, pendant qu' on note respectivement, pour les mêmes paramètres, $6.10^{1} \mathrm{UFC} / \mathrm{g} ; 5.10^{1} \mathrm{UFC} / \mathrm{g}$; moins de $10 \mathrm{UFC} / \mathrm{g}$ et $2.10^{1} \mathrm{UFC} / \mathrm{g}$ en ce qui concerne les Wagashi rouges. Il y a une diminution des charges microbiennes au niveau des Wagashi colorés en général de l'ordre décimal pour les flores totale et lactique ainsi que les levures et moisissures. Ceci justifie l'action de la chaleur produite lors de la coloration des fromages sur la destruction des microorganismes et le respect des règles d'hygiènes au cours de la production des Wagashi. Cependant, selon AFNOR (1994), la charge (UFC/g) maximale de germes aérobies mésophiles totaux tolérable pour les fromages à pâte molle pasteurisée est de $10^{3} \mathrm{UFC} / \mathrm{g}$. Les fromages séchés respectent bien cette norme. Pour les autres types de germes (lactobacilles, entérobactéries, levures et moisissures), la charge (UFC/g) maximale admise est de l'ordre de $10^{2} \mathrm{UFC} / \mathrm{g}$. En se référant à AFNOR (1994), les fromages issus du traitement thermique appliqué au cours de cette étude sont de bonne qualité microbiologique.

Tableau I : Effet du séchage sur la charge microbienne (CFU/g) des fromages

\begin{tabular}{lcccc}
\hline Types de fromages & GAMT (UFC/g) & $\begin{array}{c}\text { Lactobacilles } \\
(\mathbf{U F C} / \mathbf{g})\end{array}$ & $\begin{array}{c}\text { Entérobactéries } \\
(\mathbf{U F C} / \mathbf{g})\end{array}$ & $\begin{array}{c}\text { Levures et } \\
\text { moisissures } \\
(\mathbf{U F C} / \mathbf{g})\end{array}$ \\
\hline Fromage blanc frais & $3,2.10^{5 \mathrm{a}}$ & $2,0.10^{4 \mathrm{a}}$ & $1,2.10^{4 \mathrm{a}}$ & $1,5.10^{5 \mathrm{a}}$ \\
Fromage rouge frais & $2,8.10^{5 \mathrm{a}}$ & $1,6.10^{4 \mathrm{a}}$ & $1,0.10^{4 \mathrm{a}}$ & $1,0.10^{5 \mathrm{a}}$ \\
Fromage blanc séché & $2,5.10^{2 \mathrm{~b}}$ & $5,7.10^{1 \mathrm{~b}}$ & $4,3.10^{1 \mathrm{~b}}$ & $1,4.10^{2 \mathrm{~b}}$ \\
Fromage rouge séché & $2,1.10^{2 \mathrm{~b}}$ & $5,3.10^{1 \mathrm{~b}}$ & $3,8.10^{1 \mathrm{~b}}$ & $1,1.10^{2 \mathrm{~b}}$ \\
\hline
\end{tabular}

Les chiffres portant différentes lettres dans la même colonne sont significativement différents au seuil de 5\% 


\section{Effet du séchage sur les caractéristiques physico-chimiques des fromages}

Le tableau II présente les caractéristiques physico-chimiques des fromages blancs et rouges et met en évidence l'impact du séchage sur ces caractéristiques. Globalement, il ressort de l'analyse du tableau que les fromages frais (rouge et blanc) présentent des caractéristiques physicochimiques similaires et de même, les fromages séchés (rouge et blanc) présentent également, entre eux, des caractéristiques physico-chimiques similaires ( $\mathrm{p} \geq 0,05)$. Mais les caractéristiques physico-chimiques des fromages frais (rouge et blanc) diffèrent $(p<0,05)$ de celles des fromages séchés (rouge et blanc). Toutefois, les teneurs en eau et en lipides diffèrent $(p<0,05)$ d'un type de fromage à l'autre. Les plus fortes teneurs en eau $(57,21 \%)$ et en lipides $(22,52 \%)$ sont observées au niveau des fromages rouges frais alors que les plus faibles teneurs en ces composés sont observées au niveau des fromages blancs séchés. Les fromages séchés ont une teneur en lipides faible par rapport à celle des fromages frais. Hormis l'effet du séchage, cette différence observée entre les divers types de fromages s'expliquerait par l'effet de la coloration des fromages rouges. De plus, la réduction du taux de lipides de $2,11 \%$ pour les fromages blancs séchés et de $2,4 \%$ pour les fromages rouges séchés aurait pour explication le dégorgement d'huile observé lors du séchage. Pour ce qui est de l'eau, de forts taux de pertes en eau ont été enregistrés pour tous les types de fromage. Ces forts taux de pertes en eau seraient liés au temps prolongé de séchage (4 heures) et à la température de séchage $\left(60^{\circ} \mathrm{C}\right)$. Aussi, le barème de séchage préserve, dans une certaine mesure, la valeur nutritive des fromages car les fromages séchés et les fromages frais présentent des valeurs nutritives assez proches. Cette même observation a été faite par Buyse (2001) qui a trouvé des résultats semblables sur les fruits et légumes séchés et affirment que les aliments séchés à une température entre $35^{\circ} \mathrm{C}$ et $50^{\circ} \mathrm{C}$ préservent toute leur vitalité. Par contre, l'analyse statistique des résultats obtenus par Amegnoin (2012), portant sur l'étude de l'influence du séchage convectif à air chaud sur le fromage peulh (Wagashi) au Bénin, montre de façon globale une ressemblance des teneurs en sucre et en cendre et une grande variabilité des autres paramètres au niveau des échantillons de Wagashi frais. Quant aux Wagashi séchés, outre la teneur en huile, on ne note pas une différence significative entre les paramètres physico-chimiques. Par ailleurs, les teneurs en matière sèche et en cendres des Wagashi séchés sont supérieures à celles des Wagashi frais.

Tableau II : Effet du séchage sur la composition physico-chimique des fromages blancs et rouges

\begin{tabular}{lcccc}
\hline $\begin{array}{c}\text { Paramètres physico- } \\
\text { chimiques }\end{array}$ & $\begin{array}{c}\text { Fromage } \\
\text { blanc frais }\end{array}$ & $\begin{array}{c}\text { Fromage } \\
\text { blanc séché }\end{array}$ & $\begin{array}{c}\text { Fromage rouge } \\
\text { frais }\end{array}$ & $\begin{array}{c}\text { Fromage } \\
\text { rouge } \\
\text { séché }\end{array}$ \\
\hline Teneur en eau (\%) & $55,63 \pm 0,6^{\mathrm{a}}$ & $32,26 \mathrm{~b} \pm 0,4^{\mathrm{b}}$ & $57,21 \pm 0,2^{\mathrm{c}}$ & $33,21 \pm 0,3^{\mathrm{d}}$
\end{tabular}


pH

Acidité titrable (\% acide lactique)

Teneur en protéines $(\%$ MS)

Teneur en lipides (\% MS)

Teneur en cendres (\% MS)

Sucres totaux (\% MS)

Sucres réducteurs (\% MS)

$$
\begin{array}{ll}
5,4 \pm 0,01^{\mathrm{a}} & 5,6 \pm 0,02^{\mathrm{a}} \\
1,52 \pm 0,1^{\mathrm{a}} & 1,38 \pm 0,3^{\mathrm{a}}
\end{array}
$$

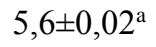

$28,32 \pm 0,11^{\mathrm{a}}$

$28,02 \pm 0,12^{\mathrm{a}}$

$27,56 \pm 0,3^{\mathrm{b}}$

$27,46 \pm 0,5^{\mathrm{b}}$

Les chiffres portant différentes lettres sur la même ligne sont significativement différents au seuil de $5 \%$.

\section{Couleur et texture des fromages frais et des fromages séchés}

Les analyses ont révélé qu'au niveau des fromages blancs, la couleur des fromages frais est plus proche de celle de la céramique blanche avec une différence totale de couleur de 27,6 $\pm 0,03$ contre $31,5 \pm 0,03$ pour les fromages blancs séchés. Cette situation pourrait s'expliquer par la formation de carapace lors du traitement thermique et le dégorgement d'huile observé. Pour les fromages rouges, les fromages séchés sont plus foncés $(\Delta \mathrm{E}: 41,2)$ que les fromages frais $(\Delta \mathrm{E}: 34,4)$. Cela pourrait s'expliquer par le fait que la déshydratation aurait engendré une augmentation de la concentration du colorant au sein des fromages. Pour ce qui est de la texture, les fromages frais sont 2 fois moins fermes et 3 fois plus friables que les fromages séchés. Ce grand écart de texture des fromages explique l'efficacité du séchage appliqué. Un tel raffermissement des fromages favoriserait le transport, la manutention et la vente dans le circuit de commercialisation car la quantité d'eau éliminée diminuerait les charges liées au poids des fromages et augmenterait la résistance au choc et à l'effritement. Shaffer (1998) explique que les aliments séchés sont faciles à transporter à cause de leur poids léger et de leur taille compressée.

\section{Effets du séchage sur la qualité sensorielle des fromages}

Le tableau III présente l'effet du traitement thermique sur la qualité sensorielle des fromages appréciée par un panel de dégustateurs. De l'analyse de ce tableau, il ressort que ce traitement affecte toutes les caractéristiques organoleptiques du fromage sauf l'odeur qui est restée presque la même. Les fromages blancs séchés sont plus jaunâtres, soit une note de 1,62 contre 3,76 pour les fromages blancs frais. De même les fromages rouges séchés sont devenus plus foncés, soit une note de 1,57 contre 2,84 pour les fromages rouges frais. On peut donc affirmer que les consommateurs préfèrent des 
fromages à coloration claire. Ces différentes observations faites par les dégustateurs confirment les analyses instrumentales au chromamètre réalisées au laboratoire. Les fromages séchés sont plus appréciés par les dégustateurs, ce que traduisent les notes d'acceptabilité globale respectives de 1,68 et 1,55 pour les fromages blancs et rouges séchés contre 0,33 et 0,45 respectivement pour les fromages blancs frais et rouges frais. La plupart de ces dégustateurs affirment que la qualité sensorielle des fromages traités est similaire à celle des fromages frits qu'ils ont l'habitude de consommer et qu'ils apprécient. Ils ont souligné que les fromages rouges ont un goût légèrement amer. Cet état de chose peut être dû à l'effet du traitement thermique sur certains composés du fromage qui se seraient dénaturés.

Tableau III. Effets du séchage sur la qualité sensorielle des fromages

\begin{tabular}{|c|c|c|c|c|c|c|}
\hline \multirow[b]{2}{*}{$\begin{array}{l}\text { Types de } \\
\text { fromages }\end{array}$} & \multirow[b]{2}{*}{$\begin{array}{c}\text { Couleu } \\
\mathbf{r}\end{array}$} & \multicolumn{2}{|c|}{ Texture } & \multirow[b]{2}{*}{ Odeur } & \multirow[b]{2}{*}{ Goût } & \multirow{2}{*}{$\begin{array}{c}\text { Accepta } \\
\text { bilité } \\
\text { globale }\end{array}$} \\
\hline & & $\begin{array}{l}\text { Friabilité } \\
(\mathbf{N})\end{array}$ & $\begin{array}{l}\text { Fermeté } \\
(\mathbf{N})\end{array}$ & & & \\
\hline $\begin{array}{l}\text { Fromage blanc } \\
\text { frais }\end{array}$ & $\begin{array}{c}3,76 \pm 0 \\
23 \mathrm{a}\end{array}$ & $1,74 \pm 0,43 \mathrm{a}$ & $\begin{array}{c}0,71 \pm 0,1 \\
6 \mathrm{a}\end{array}$ & $\begin{array}{c}1,72 \pm 0 \\
12 \mathrm{a}\end{array}$ & $\begin{array}{c}0,88 \pm 0,4 \\
1 \mathrm{a}\end{array}$ & $\begin{array}{c}0,33 \pm 0,1 \\
2 \mathrm{a}\end{array}$ \\
\hline $\begin{array}{c}\text { Fromage blanc } \\
\text { séché }\end{array}$ & $\begin{array}{c}1,62 \pm 0 \\
03 \mathrm{~b}\end{array}$ & $0,65 \pm 0,25 b$ & $\begin{array}{c}1,85 \pm 0,1 \\
4 b\end{array}$ & $\begin{array}{c}1,64 \pm 0 \\
06 \mathrm{a}\end{array}$ & $\begin{array}{c}0,76 \pm 0,2 \\
3 \mathrm{a}\end{array}$ & $\begin{array}{c}1,68 \pm 0,2 \\
5 \mathrm{~b}\end{array}$ \\
\hline $\begin{array}{l}\text { Fromage rouge } \\
\text { frais }\end{array}$ & $\begin{array}{c}2,84 \pm 0 \\
22 \mathrm{c}\end{array}$ & $1,81 \pm 0,52 \mathrm{a}$ & $\begin{array}{c}0,66 \pm 0,2 \\
1 \mathrm{a}\end{array}$ & $\begin{array}{c}1,87 \pm 0 \\
52 \mathrm{a}\end{array}$ & $\begin{array}{c}0,82 \pm 0,1 \\
3 \mathrm{a}\end{array}$ & $\begin{array}{c}0,45 \pm 0,1 \\
5 \mathrm{a}\end{array}$ \\
\hline $\begin{array}{c}\text { Fromage rouge } \\
\text { séché }\end{array}$ & $\begin{array}{c}1,57 \pm 0 \\
12 \mathrm{~b}\end{array}$ & $0,58 \pm 0,15 b$ & $\begin{array}{c}1,83 \pm 0,1 \\
4 b\end{array}$ & $\begin{array}{c}1,63 \pm 0 \\
42 \mathrm{a}\end{array}$ & $\begin{array}{c}0 \\
, 77 \pm 0,32 \mathrm{a}\end{array}$ & $\begin{array}{c}1,55 \pm 0,2 \\
3 \mathrm{~b}\end{array}$ \\
\hline
\end{tabular}

Les chiffres portant les mêmes lettres dans la même colonne ne sont pas significativement différents au seuil de $5 \%$.

\section{Effet combiné du séchage et de conditionnement sous vide sur la qualité microbiologique des fromages}

Les différents types de fromages séchés ont été conditionnés dans un emballage sous vide et conservés au réfrigérateur à et la température ambiante afin d'observer le comportement des fromages dans ces conditions lors de la conservation. Le tableau IV présente l'évolution des caractéristiques microbiologiques durant la conservation des fromages séchés, emballés sous vide et conservés au réfrigérateur entre $4-5^{\circ} \mathrm{C}$. De l'analyse du tableau IV, il ressort que les charges microbiennes des fromages mis sous vide et conservés au réfrigérateur demeurent plus ou moins constantes dans le temps. Il n'y a pas de différence significative $(\mathrm{p} \geq 0,05)$ entre les charges enregistrées. Ainsi, la réfrigération stabilise davantage le fromage et empêche sa détérioration. Ces résultats confirment les observations faites par Foucaud (2006); Vierling (1998) et Spreer (1998) sur les produits alimentaires réfrigérés. Pour ces auteurs, la température basse ralentit le développement de la plupart des microbes dangereux, mais il faut souligner que l'effet obtenu ici serait une combinaison des actions du vide et de la réfrigération car les fromages 
témoins, c'est-à-dire non conditionnés sous vide et conservés par réfrigération à $4-5^{\circ} \mathrm{C}$ ont présenté dix (10) jours après, des signes de détérioration, notamment l'aspect gluant, l'odeur putride et l'apparition de touffes noirâtres à la surface du fromage, traduisant l'infestation par les moisissures.

Tableau IV : Évolution de la charge (UFC/g) microbienne des fromages séchés, conditionnés sous vide et réfrigérés à $4-5^{\circ} \mathrm{C}$

\begin{tabular}{|c|c|c|c|c|c|c|c|c|}
\hline \multirow{2}{*}{$\begin{array}{c}\text { Durée de } \\
\text { conservatio } \\
\text { n }\end{array}$} & \multicolumn{2}{|c|}{ GAMT } & \multicolumn{2}{|c|}{ Lactobacilles } & \multicolumn{2}{|c|}{ Entérobactéries } & \multicolumn{2}{|c|}{$\begin{array}{l}\text { Levures et } \\
\text { moisissures }\end{array}$} \\
\hline & FBSR* & $\underset{*}{\text { FRSR }^{*}}$ & FBSR & FRSR & FBSR & FRSR & FBSR & FRSR \\
\hline T0 & $\begin{array}{c}2,5.10^{2} \\
a\end{array}$ & $2,1.10^{2 b}$ & $5,7.10^{1 \mathrm{a}}$ & $\begin{array}{l}5,3 . \\
10^{1 \mathrm{~b}}\end{array}$ & $4,3 \cdot 10^{1 \mathrm{a}}$ & $3,8.10^{1 \mathrm{~b}}$ & $1,4 \cdot 10^{2 \mathrm{~d}}$ & $1,1.10^{2 \mathrm{a}}$ \\
\hline T1 & $2,4 \cdot 10^{2 \mathrm{a}}$ & $2,0.10^{2 \mathrm{~b}}$ & $5,4.10^{1 \mathrm{a}}$ & $\begin{array}{l}5,1 \\
10^{1 \mathrm{~b}}\end{array}$ & $4,1.10^{1 \mathrm{a}}$ & $3,8 \cdot 10^{1 \mathrm{~b}}$ & $1,2.10^{2 \mathrm{~d}}$ & $1,0.10^{2 \mathrm{a}}$ \\
\hline $\mathbf{T 2}$ & $2,3 \cdot 10^{2 a}$ & $1,8.10^{2 \mathrm{~b}}$ & $5,3.10^{1 \mathrm{a}}$ & $\begin{array}{l}5,0 . \\
10^{1 \mathrm{~b}}\end{array}$ & $4.10^{1 \mathrm{a}}$ & $3,4 \cdot 10^{1 \mathrm{~b}}$ & $1,1 \cdot 10^{2 \mathrm{~d}}$ & $1,0.10^{2 \mathrm{a}}$ \\
\hline
\end{tabular}

Les chiffres portant les mêmes lettres dans la même colonne ne sont pas significativement différents au seuil de 5\%.*FBSR : Fromage blanc séché emballé sous vide et réfrigéré (4-5

$\left.{ }^{\circ} \mathrm{C}\right)$ et $* *$ FRSR : Fromage rouge séché emballé sous vide et réfrigéré $\left(4-5^{\circ} \mathrm{C}\right)$

Par ailleurs, le tableau V présente les caractéristiques microbiologiques durant la conservation des fromages séchés, emballés sous vide et conservés à la température ambiante entre $28-32{ }^{\circ} \mathrm{C}$. Dans ces conditions, une évolution à la hausse des germes aérobies mésophiles totaux, des lactobacilles et des levures et moisissures est enregistrée alors que le nombre d'entérobactérie s'annule dans les deux types de fromage (rouges et blancs) déjà après un mois de conservation. Cette évolution pourrait s'expliquer par le fait que le vide ne serait pas absolu. Aussi, les aliments conservés sous vide ne sont pas dépourvus de microorganismes; et c'est pourquoi il est important de les maintenir à la réfrigération (Sacramento, 2008). Par contre, la disparition des entérobactéries s'expliquerait par le développement des lactobacilles qui baissent le $\mathrm{pH}$ et augmentent l'acidifié du milieu rendant impossible la survie de ces entérobactéries dont la population était déjà faible dans le fromage au début des expériences. Vignola (2002) a fait les mêmes observations sur les produits alimentaires transformés. Pour cet auteur, les bactéries lactiques inhibent souvent le développement des entérobactéries dans un aliment. Enfin, l'augmentation du nombre de levures et moisissures est en rapport avec celles des bactéries lactiques. Ceci a été confirmé par Mounirou (1990) qui a noté que l'évolution croissante des levures et moisissures et des bactéries lactiques traduisant la symbiose entre les bactéries lactiques et les levures.

Tableau V : Évolution de la charge (CFU/g) microbienne des fromages séchés, conditionné sous vide et conservés à la température ambiante
GAMT
Lactobacilles
Entérobactéries
Levures et moisissures 


\begin{tabular}{ccccccccc}
$\begin{array}{c}\text { Durée de } \\
\text { conservat } \\
\text { ion }\end{array}$ & $\begin{array}{c}\text { FBST } \\
\mathbf{A}^{*}\end{array}$ & $\begin{array}{c}\text { FRST } \\
\mathbf{A}^{* *}\end{array}$ & FBSTA & $\begin{array}{c}\text { FRST } \\
\mathbf{A}\end{array}$ & $\begin{array}{c}\text { FBST } \\
\mathbf{A}\end{array}$ & $\begin{array}{c}\text { FRST } \\
\mathbf{A}\end{array}$ & FBSTA & $\begin{array}{c}\text { FRST } \\
\mathbf{A}\end{array}$ \\
\hline T0 & $2,5.10$ & $2,1.102$ & $5,7.101 \mathrm{a}$ & $5,3$. & $4,3.10$ & $3,8.10$ & $1,4.102$ & $1,1.10$ \\
& $2 \mathrm{a}$ & $\mathrm{a}$ & & $101 \mathrm{~b}$ & $1 \mathrm{a}$ & $1 \mathrm{~b}$ & $\mathrm{a}$ & $2 \mathrm{a}$ \\
T1 & $2,6.10$ & $1,8.103$ & $3,2.104$ & $5,1$. & 0 & 0 & $1,5$. & $1,3$. \\
& $3 \mathrm{~b}$ & $\mathrm{~b}$ & $\mathrm{~b}$ & $101 \mathrm{~b}$ & & & $103 \mathrm{~b}$ & $103 \mathrm{~b}$ \\
T2 & $1,7.10$ & $1,2.104$ & $2,4.105 \mathrm{c}$ & $5,0$. & 0 & 0 & $1,2.104$ & $1,0.10$ \\
& $4 \mathrm{c}$ & $\mathrm{c}$ & & $101 \mathrm{~b}$ & & & $\mathrm{c}$ & $4 \mathrm{c}$ \\
\hline
\end{tabular}

Les chiffres portant les mêmes lettres dans la même colonne ne sont pas significativement différents au seuil de 5\%.*FBSTA : Fromage blanc séché emballé sous vide et conservé à température ambiante $\left(28-32^{\circ} \mathrm{C}\right)$ et $* *$ FRSTA : Fromage rouge séché emballé sous vide et conservé à température ambiante $\left(28-32^{\circ} \mathrm{C}\right)$

\section{Évolution des caractéristiques physico-chimiques des fromages stabilisés et conditionnés sous vide}

A la température de $4-5^{\circ} \mathrm{C}$, les caractéristiques physico-chimiques des fromages réfrigérés sont restées presque constantes dans le temps. Ces résultats sont en relation directe avec les résultats microbiologiques obtenus. La non-prolifération excessive des microorganismes dans les fromages réfrigérés peut expliquer cet état de chose. À cet effet, Jouan (2002) explique que la vitesse de prolifération des microorganismes dans un aliment traduit celle de dégradation de cet aliment. Le tableau VI présente l'évolution des caractéristiques physico-chimiques des fromages séchés, mis sous vide et conservés à la température ambiante $\left(28-32^{\circ} \mathrm{C}\right)$ : la teneur en eau et l'acidité titrable augmentent dans le temps. Les autres paramètres baissent de façon continuelle. Dans l'ensemble, ces résultats s'expliquent par la prolifération importante des germes aérobies totaux, des bactéries lactiques et des levures et moisissures dans les fromages considérés. L'augmentation de la teneur en eau entraîne une baisse de la matière sèche. L'augmentation de la teneur en eau résulterait de l'hydrolyse des composés tels que les protéines, les glucides et les lipides contenus dans les fromages. Des résultats semblables ont été trouvés par Akissoe (1992) sur les produits fermentés, notamment le mawè, une pâte humide à base de farine de maïs consommé au Bénin.

Tableau VI : Évolution des caractéristiques physico-chimiques des fromages séchés, mis sous vide et conservés à la température ambiante $\left(28-32^{\circ} \mathrm{C}\right)$

\begin{tabular}{ccccccc}
\hline & \multicolumn{6}{c}{ Durée de conservation } \\
\cline { 2 - 7 } Caractéristiques & \multicolumn{2}{c}{ T0 } & \multicolumn{2}{c}{ T1 } & \multicolumn{2}{c}{ T2 } \\
\cline { 2 - 7 } & FBSTA* & FRSTA & \multirow{2}{*}{ FBSTA } & FRSTA & FBSTA & FRSTA \\
\hline Teneur en eau (\%) & $32,26 \pm 0,52$ & $33,21 \pm 0$, & $35,03 \pm 0$, & $35,25 \pm 0$, & $38,12 \pm 0$, & $37,5 \pm 0,2$ \\
pH & $\mathrm{a}$ & $3 \mathrm{c}$ & $45 \mathrm{~b}$ & $21 \mathrm{~b}$ & $48 \mathrm{c}$ & $5 \mathrm{a}$ \\
& $5,4 \pm 0,01 \mathrm{a}$ & $6,3 \pm 0,05$ & $5,02 \pm 0,0$ & $5,7 \pm 0,04$ & $4,81 \pm 0,0$ & $5,2 \pm 0,06$ \\
& & $\mathrm{c}$ & $4 \mathrm{~b}$ & $\mathrm{~b}$ & $2 \mathrm{c}$ & $\mathrm{a}$
\end{tabular}




\begin{tabular}{ccccccc} 
Acidité titrable $(\%$ & $1,52 \pm 0,14 \mathrm{a}$ & $0,24 \pm 0,1$ & $2,02 \pm 0,1$ & $1,41 \pm 0,1$ & $2,41 \pm 0,1$ & $1,56 \pm 0,2$ \\
acide lactique) & $1 \mathrm{c}$ & $6 \mathrm{~b}$ & $\mathrm{~b}$ & $2 \mathrm{c}$ & $\mathrm{a}$ \\
Teneur en protéines & $28,02 \pm 2,35$ & $27,46 \pm 1$, & $23,31 \pm 2$, & $25,34 \pm 1$, & $18,01 \pm 2$, & $21,3 \pm 1,0$ \\
(\% MS) & $\mathrm{a}$ & $02 \mathrm{c}$ & $2 \mathrm{~b}$ & $04 \mathrm{~b}$ & $05 \mathrm{c}$ & $6 \mathrm{a}$ \\
Teneur en lipides $(\%$ & $19,15 \pm 3,41$ & $20,48 \pm 2$, & $17,1 \pm 3,2$ & $17,42 \pm 2$, & $14,8 \pm 3,5$ & $17,15 \pm 2$, \\
MS) & $\mathrm{a}$ & $52 \mathrm{c}$ & $3 \mathrm{~b}$ & $48 \mathrm{~b}$ & $3 \mathrm{c}$ & $5 \mathrm{a}$ \\
Teneur en cendres & $5,01 \pm 0,3 \mathrm{a}$ & $3,53 \pm 0,1$ & $4,0 \pm 0,02$ & $2,45 \pm 0,2$ & $2,96 \pm 0,4$ & $1,43 \pm 0,1$ \\
(\% MS) & & $2 \mathrm{a}$ & $\mathrm{b}$ & $5 \mathrm{~b}$ & $7 \mathrm{c}$ & $8 \mathrm{c}$ \\
Sucres totaux $(\%$ & $15,36 \pm 0,01$ & $16,13 \pm 0$, & $12,8 \pm 0,0$ & $14,03 \pm 0$, & $9,5 \pm 0,35$ & $11,22 \pm 0$, \\
MS) & $\mathrm{a}$ & $32 \mathrm{c}$ & $3 \mathrm{~b}$ & $28 \mathrm{~b}$ & $\mathrm{c}$ & $25 \mathrm{a}$ \\
Sucres réducteurs & $10,85 \pm 0,2 \mathrm{a}$ & $11,48 \pm 1$, & $8,36 \pm 0,6$ & $9,17 \pm 1,1$ & $5,85 \pm 0,3$ & $7,11 \pm 1,2$ \\
(\% MS) & & $05 \mathrm{c}$ & $1 \mathrm{~b}$ & $\mathrm{~b}$ & $2 \mathrm{c}$ & $2 \mathrm{a}$ \\
\hline
\end{tabular}

Les chiffres portant les mêmes lettres sur la même ligne ne sont pas significativement différents au seuil de 5\%. *FBSTA : Fromage blanc séché emballé sous vide et conservé à température ambiante $\left(28-32^{\circ} \mathrm{C}\right)$ et $* *$ FRSTA : Fromage rouge séché emballé sous vide et conservé à température ambiante $\left(28-32^{\circ} \mathrm{C}\right)$.

\section{Évolution de la qualité sensorielle des fromages séchés et conditionnés sous vide}

Il découle de l'analyse du tableau VII que les fromages gardés à la réfrigération conservent plus longtemps leur qualité sensorielle que les fromages conservés à température ambiante. Les fromages rouges et blancs conservés à la température ambiante s'acidifient au cours du temps. Les consommateurs acceptent de moins en moins au cours du temps ces fromages. Il en est de même pour les fromages rouges réfrigérés dont le goût devient légèrement amer après deux mois. En définitive, les consommateurs n'apprécient que les fromages blancs réfrigérés. La plupart des observations faites par les dégustateurs sont en rapport avec les résultats trouvés lors des analyses physico- chimiques.

Tableau VII : Évolution de la qualité sensorielle des fromages séchés et mis sous vide

\begin{tabular}{|c|c|c|c|c|c|c|c|}
\hline \multirow{2}{*}{$\begin{array}{c}\text { Durée de } \\
\text { conservati } \\
\text { on }\end{array}$} & \multirow[b]{2}{*}{$\begin{array}{l}\text { Types de } \\
\text { fromage }\end{array}$} & \multirow[b]{2}{*}{$\begin{array}{c}\text { Couleu } \\
\mathbf{r}\end{array}$} & \multicolumn{2}{|c|}{ Texture } & \multirow[b]{2}{*}{ Odeur } & \multirow[b]{2}{*}{ Goût } & \multirow[b]{2}{*}{$\begin{array}{l}\text { Acceptabil } \\
\text { ité globale }\end{array}$} \\
\hline & & & $\begin{array}{l}\text { Friabilité } \\
(\mathbf{N})\end{array}$ & $\begin{array}{c}\text { Fermeté } \\
(\mathbf{N})\end{array}$ & & & \\
\hline \multirow{4}{*}{ T0 } & FBSR* & $\begin{array}{c}1,62 \pm 0 \\
03 \mathrm{a}\end{array}$ & $\begin{array}{c}0,65 \pm 0,2 \\
5 \mathrm{a}\end{array}$ & $1,85 \pm 0,14 \mathrm{a}$ & $\begin{array}{c}1,64 \pm 0 \\
06 \mathrm{a}\end{array}$ & $\begin{array}{c}0,76 \pm 0,2 \\
3 a\end{array}$ & $1,68 \pm 0,25 \mathrm{a}$ \\
\hline & $\underset{*}{\text { FBSTA* }}$ & $\begin{array}{c}1,62 \pm 0 \\
03 a\end{array}$ & $\begin{array}{c}0,65 \pm 0,2 \\
5 \mathrm{a}\end{array}$ & $1,85 \pm 0,14 \mathrm{a}$ & $\begin{array}{c}1,64 \pm 0 \\
06 \mathrm{a}\end{array}$ & $\begin{array}{c}0,76 \pm 0,2 \\
3 a\end{array}$ & $1,68 \pm 0,25 \mathrm{a}$ \\
\hline & FRSR** & $\begin{array}{c}1,57 \pm 0 \\
12 \mathrm{a}\end{array}$ & $\begin{array}{c}0,58 \pm 0,1 \\
5 \mathrm{a}\end{array}$ & $\begin{array}{c}0 \\
, 77 \pm 0,32 \mathrm{a}\end{array}$ & $\begin{array}{c}1,63 \pm 0 \\
42 \mathrm{a}\end{array}$ & $\begin{array}{c}0 \\
, 77 \pm 0,32 \mathrm{a}\end{array}$ & $1,55 \pm 0,23 \mathrm{a}$ \\
\hline & $\underset{* * *}{\text { FRSTA* }}$ & $\begin{array}{c}1,57 \pm 0 \\
12 \mathrm{a} \\
\end{array}$ & $\begin{array}{c}0,58 \pm 0,1 \\
5 \mathrm{a} \\
\end{array}$ & $1,83 \pm 0,14 \mathrm{a}$ & $\begin{array}{c}1,63 \pm 0 \\
42 \mathrm{a} \\
\end{array}$ & $\begin{array}{c}0 \\
, 77 \pm 0,32 \mathrm{a} \\
\end{array}$ & $1,55 \pm 0,23 \mathrm{a}$ \\
\hline \multirow{2}{*}{ T1 } & FBSR* & $\begin{array}{c}1,56 \pm 0 \\
05 \mathrm{a}\end{array}$ & $\begin{array}{c}0,63 \pm 0,0 \\
2 \mathrm{a}\end{array}$ & $1,87 \pm 0,36 \mathrm{a}$ & $\begin{array}{c}1,66 \pm 0 \\
18 \mathrm{a}\end{array}$ & $\begin{array}{c}0,77 \pm 0,0 \\
5 \mathrm{a}\end{array}$ & $1,67 \pm 0,03 a$ \\
\hline & $\underset{*}{\text { FBSTA* }}$ & $\begin{array}{c}1,53 \pm 0 \\
42 \mathrm{a}\end{array}$ & $\begin{array}{c}1,62 \pm 0,1 \\
4 \mathrm{~b}\end{array}$ & $0,85 \pm 0,05 \mathrm{~b}$ & $\begin{array}{c}0,60 \pm 0 \\
21 \mathrm{~b}\end{array}$ & $\begin{array}{c}1,76 \pm 0,1 \\
3 b\end{array}$ & $0,34 \pm 0,12 b$ \\
\hline
\end{tabular}




\begin{tabular}{|c|c|c|c|c|c|c|c|}
\hline & FRSR** & $\begin{array}{c}1,55 \pm 0, \\
3 \mathrm{a}\end{array}$ & $\begin{array}{c}0,57 \pm 0,2 \\
5 \mathrm{a}\end{array}$ & $1,78 \pm 0,35 \mathrm{a}$ & $\begin{array}{c}1,65 \pm 0, \\
15 \mathrm{a}\end{array}$ & $\begin{array}{c}0,74 \pm 0,1 \\
2 \mathrm{a}\end{array}$ & $1,12 \pm 0,6 \mathrm{a}$ \\
\hline & $\underset{* * *}{\operatorname{FRSTA}}$ & $\begin{array}{c}1,55 \pm 0, \\
12 \mathrm{a}\end{array}$ & $\begin{array}{c}1,61 \pm 0,4 \\
3 \mathrm{~b}\end{array}$ & $0,75 \pm 0,41 b$ & $\begin{array}{c}0,52 \pm 0 \\
05 \mathrm{~b}\end{array}$ & $\begin{array}{c}1,51 \pm 0,2 \\
4 \mathrm{~b}\end{array}$ & $0,23 \pm 0,02 b$ \\
\hline \multirow{4}{*}{ T3 } & FBSR* & $\begin{array}{c}1,55 \pm 0, \\
03 \mathrm{a}\end{array}$ & $\begin{array}{c}0,71 \pm 0,1 \\
5 a\end{array}$ & $1,84 \pm 0,26 \mathrm{a}$ & $\begin{array}{c}1,67 \pm 0 \\
04 \mathrm{a}\end{array}$ & $\begin{array}{c}0,75 \pm 0,1 \\
6 \mathrm{a}\end{array}$ & $1,74 \pm 0,01 \mathrm{a}$ \\
\hline & $\underset{*}{\text { FBSTA }} *$ & $\begin{array}{c}1,68 \pm 0, \\
24 \mathrm{a}\end{array}$ & $\begin{array}{c}1,72 \pm 0,1 \\
8 b\end{array}$ & $0,82 \pm 0,23 b$ & $\begin{array}{c}0,64 \pm 0 \\
23 b\end{array}$ & $\begin{array}{c}1,78 \pm 0,0 \\
4 \mathrm{~b}\end{array}$ & $0,28 \pm 0,04 \mathrm{~b}$ \\
\hline & FRSR $* *$ & $\begin{array}{c}1,57 \pm 0, \\
33 \mathrm{a}\end{array}$ & $\begin{array}{c}0,58 \pm 0,0 \\
3 a\end{array}$ & $1,65 \pm 0,51 \mathrm{a}$ & $\begin{array}{c}1,68 \pm 0, \\
32 \mathrm{a}\end{array}$ & $\begin{array}{c}0,72 \pm 0,0 \\
2 \mathrm{a}\end{array}$ & $1,10 \pm 0,02 \mathrm{a}$ \\
\hline & $\underset{* * *}{\text { FRSTA }} *$ & $\begin{array}{c}1,63 \pm 0, \\
46 \mathrm{a}\end{array}$ & $\begin{array}{c}1,65 \pm 0,1 \\
3 b\end{array}$ & $0,72 \pm 0,02 b$ & $\begin{array}{c}0,58 \pm 0, \\
24 b\end{array}$ & $\begin{array}{c}1,58 \pm 0,2 \\
2 b\end{array}$ & $0,21 \pm 0,01 b$ \\
\hline
\end{tabular}

Les chiffres portant les mêmes lettres dans la même colonne ne sont pas significativement différents au seuil de 5\%. *FBSR : Fromage blanc séché emballé sous vide et réfrigéré (4-5

$\left.{ }^{\circ} \mathrm{C}\right)$; ** FBSTA : Fromage blanc séché emballé sous vide et conservé à température ambiante $\left(28-32{ }^{\circ} \mathrm{C}\right) ; * * *$ FRSR : Fromage rouge séché emballé sous vide et réfrigéré (4-5

$\left.{ }^{\circ} \mathrm{C}\right)$; **** FRSTA : Fromage rouge séché emballé sous vide et conservé à température ambiante $\left(28-32{ }^{\circ} \mathrm{C}\right)$.

\section{Rentabilité économique de la production du fromage peulh conditionné sous vide seuil de rentabilité}

La quantité de fromage produite par an dans les conditions de l'expérimentation serait de 34.800 sachets, soit 116 sachets par production. Le coût d'opération s'élève à 1.868 .100 FCFA et les charges de structure ou amortissement du matériel utilisé sont de 58142,83 FCFA. Le seuil de rentabilité de la production de fromage conditionné sous vide s'évalue donc comme suit : $1.868 .100 \mathrm{y} / 34.800=53,68$ pour y sachets. Le prix de vente d'un sachet de fromage conservé sous vide étant de 150 FCFA, on aura : 53,68y $+58142,83=150 \mathrm{y} ; \mathrm{y}=1083$ (1083 sachets) ; soit $54150 \mathrm{~g}$ de fromage ou 54,15 $\mathrm{kg}$ de fromage conservé sous vide. Donc, pour que cette unité puisse couvrir ses charges de production, elle doit livrer au moins 1083 sachets par an de fromages conditionnés sous vide à 150 FCFA l'unité.

\section{Marge bénéficiaire unitaire}

Le coût de production des 34.800 sachets de fromages conditionnés sous vide revient donc à 1926242,8 FCFA; soit 55,35 FCFA / sachet. Si le sachet est vendu à 150 FCFA, la marge brute serait de 94,65 FCFA soit $63,1 \%$ par sachet. Les frais de commercialisation étant de 240.000 FCFA soit 6,90 FCFA/ sachet, la marge nette revient à 87,75 FCFA ; soit 58,5\%.

\section{Résultat d'exploitation annuel}

Pour une année entière couvrant 300 jours de production, la vente de 34.800 sachets donnera une recette de 5.220.000 FCFA étant donné que le prix d'un sachet est de 150 FCFA. Les charges variables équivalentes s'élèvent à 
1.868.100 FCFA, le résultat d'exploitation donnera donc 5.220.000 $(1.868 .100+58142,83)$, soit 3.293.757,2 FCFA.

\section{Conclusion et suggestions}

La méthode traditionnelle de conservation du fromage peulh demeure contraignante, engendre de lourdes pertes en fromage et en nutriments et, tout en exposant le consommateur à des risques d'intoxication alimentaire, constitue un frein pour la commercialisation et la promotion de ce produit. Pour passer de l'étape actuelle de la technologie traditionnelle à une production semi industrielle ou industrielle, il urge de mettre au point des techniques modernes de conservation faciles d'accès et s'adaptant aux réalités socio- économiques et culturelles des consommateurs. La conservation sous vide répond bien à cette exigence étant donné qu'elle nécessite très peu de moyens et que les prix des produits qui en sont issus concurrencent bien ceux des fromages rencontrés sur les marchés locaux. Cette étude a ouvert une nouvelle piste de recherche en testant l'influence du conditionnement sous vide sur l'aptitude à la conservation des fromages peulh. Les caractéristiques physico-chimiques sont globalement conservées surtout quand les fromages mis sous vide sont réfrigérés. La déshydratation thermique appliquée avant la mise sous vide permet de réduire de plus de la moitié la charge microbienne $\mathrm{du}$ fromage frais. Ce séchage, tout en diminuant la charge microbienne du fromage, a permis aussi la préservation de sa qualité nutritionnelle et sensorielle. Enfin, pour une maîtrise totale de ce nouveau procédé, de nouvelles recherches doivent être entreprises afin de déterminer la date limite de consommation des fromages ainsi conditionnés sous vide.

\section{References:}

1. AACC, 1984. Approved methods of the American Association of Cereal Chemists. $8^{\text {th }}$ Edition, St Paul, MN, USA.

2. AFNOR, 1994. Critères microbiologiques sur les fromages au lait cru ou au lait thermisé. Arrêté du 30 mars 1994 publié au Journal Officiel du 21 avril 1994.

3. Akissoe N.H.,1992. Influence de quatre variétés de maïs sur les caractéristiques physicochimiques du mawè, pâte fermentée traditionnelle. Thèse d'ingénieur agronome. Université d'AbomeyCalavi.

4. Amegnoin A. N., 2012. Etude de l'influence du séchage convectifà air chaud sur le fromage peulh (waragashi) au Bénin, Thèse d'ingénieur de conception en technologie alimentaire; Université d'AbomeyCalavi, Bénin, pp. 51-59. 
5. AOAC, 1984. Approved methods of the American Association of Cereal Chemists, 8th Edition St Paul, AOAC. USA.

6. Buyse Y.N.D., 2001. Le séchage des aliments, un procédé de santé. Editions logiques, pp.1-7.

7. Directive 92/46/CEE DU Conseil du 16 juin 1992 arrêtant les règles sanitaires pour la production et la mise sur le marché de lait cru, de lait traité thermiquement et de produits à base de lait.

8. Dossou J., 2004. Amélioration de la technologie traditionnelle de fabrication du fromage peulh au Bénin. Proposition de recherche. DNSA/FSA/ Université d'Abomey-Calavi, 51p.

9. Dossou J., 2015 ; Rapport d'activités recherches du projet « Improving milk productivity band value addition to enhance food security in West Africa » (IMPROMILK) ; CORAF/WECARD ; Université d'Abomey-Calavi ; Bénin 16p.

10. Dossou J., Adoté S. et A. Soulé., 2006. Fiche technique de production et transformation du lait frais en fromage peulh au Bénin. Département de Nutrition et Sciences Alimentaire, Faculté des Sciences Agronomiques, Université d'Abomey-Calavi, 33 pp.

11. Dossou J.; Atchouké G. D. ; Dabadé D. S. ; Azokpota P. et Montcho J. K., 2016. Evaluation comparative de la qualité nutritionnelle et sanitaire du lait de différentes races de vaches de quelques zones d'élevage du Bénin ; European Scientific Journal January 2016 edition vol.12, No.3 ISSN: 1857 - 7881 (Print) e - ISSN 1857- 7431 ; pp. 148 -153 .

12. Egounléty M., 1982. Stabilisation du fromage pâte molle de l'Afrique de l'ouest par traitement chimique et emballage gazeux. Thèse d'Ingénieur Agronome, Faculté des Sciences Agronomiques, Université Nationale du Bénin, Abomey-Calavi, République du Bénin. $133 \mathrm{p}$.

13. Egounléty M., Edema M., Yehouessi B. et Ahouansou E.A., 1994. Production et qualité du fromage Peulh (waragashi) en République du Bénin. Rapport de Recherche. Département de Nutrition et Sciences Alimentaire, Faculté des Sciences Agronomiques, Université Nationale duBénin, Abomey-Calavi, pp. 30-33.

14. Florez A. B., Hernandez-Barranco A. M., Marcos I., Mayo B., 2006. Biochemical and microbiological characterization of artisan kid rennet 
extracts used for Cabrales cheese manufacture.LWT 39 (2006) 605612 pp.

15. Foucaud J., 2006. Les produits marins réfrigérés. INRA Editions, $128 \mathrm{p}$.

16. Hamama A., El Mouktafi M., 1990. "Étude de la qualité hygiénique du lait cru produit au Maroc", Maghreb Vétérinaire, vol. 5,pp. 17-20.

17. Jouan P., 2002. Propriétés lytiques des microorganismes. INRA Editions, 128p.

18. Kees M., 1996. Le fromage peulh : facile à produire et bien apprécié, une technologie àvulgariser. Rapport de recherche GTZ, Université Eschborn, RFA. pp. 8-25.

19. Kèkè M., 2005. Contribution à l'amélioration de la technologie de production du fromage peulh appelé waragashi. Mémoire de maîtrise ; Faculté des Sciences et Techniques (FAST)/Université d'AbomeyCalavi.

20. Kora S., 2005. Contribution à l'amélioration technologique de fabrication du fromage peuhl : mise au point et test de performance d'un coagulant végétal à base de Calotropis procera; Thèse d'ingénieur agronome; Faculté des Sciences Agronomiques, Université d'Abomey-Calavi, Bénin, 105p.

21. Labioui H., EL Moualdi L., Benzakour A., EL Yachioui El Hassan B. M., Ouhssine M. ; 2009. « Etude physicochimique et microbiologique de laits crus »; Bull. Soc. Pharm. Bordeaux, 148, pp.7-16.

22. Mounirou O., 1990. Utilisation la flore microbienne de Ogui dans le processus de conservation du poisson par voie fermentée. Thèse d'ingénieur agronome. Université d'Abomey-Calavi.

23. Nout M. J. R, Rombouts F. M., and Havelaar A., 1989. Effect of accelerated natural lactic fermentation of infant good ingredients on some pathogenic microorganism. International Journal of Food Microbiology, vol. 8, pp. 351-361.

24. Oubari R., 2004. "Analyse physicochimique et microbiologique du lait et effet de la propolis sur la qualité microflore d'intérêt hygiénique du lait", Mémoire, Faculté des Sciences de Kénitra, Maroc. 
25. Outmani A., 2009 ; Commercialisation collective du fromage peuhl ! Quelle voie ? Rapport d'activités sur la filière lait et fromage ; SNV, Antenne Natitingou ; Benin ; $4 \mathrm{p}$.

26. Sacramento O. R. H., 2008. Contribution à l'amélioration de la conservation du Waragashi par l'effet combiné du séchage et de l'emballage sous vide. Thèse d'Ingénieur Agronome, Faculté des Sciences Agronomiques, Université d'Abomey-Calavi, République du Bénin, 74p.

27. Shaffer M., 1998. Food drying, Backwoods home magazine, 39p.

28. Spreer E. 1988; Technologie der Milchverarbeitung; VEB Fachbuchverlag, Leipzig ; DDR ; 540 p.

29. Taybi N. O., Arfaoui A., and Fadli M., 2014. Evaluation of microbiological quality of raw milk in the region of Gharb, Morocco; International Journal of Innovation and Scientific Research; ISSN 2351-8014 Vol. 9 No. 2 ; pp. 487-493.

30. Vierling E., 1998 ; Aliments et Boissons ; Technologies et aspects réglementaires ; Editions Biosciences et Techniques ; France ; 190 p.

31. Vierling E., 1998; Aliments et Boissons; Filières et produits ; Editions Biosciences et Techniques ; France ; 271 p.

32. Vignola C.L., 2002. Transformation des aliments sur le plan technique et scientifique. Presses Intern. Polytechnique Campus de l'Université, $600 \mathrm{p}$. 